\title{
Exact Solutions and Localized Structures for a $(3+1)$-Dimensional Burgers Equation
}

\author{
Y.-Z. PENG* \\ School of Mathematics and Statistics, Wuhan University, Wuhan, 430072, P.R. China
}

(Received January 6, 2011)

\begin{abstract}
A $(3+1)$-dimensional Burgers equation is studied by the singular manifold method. By choosing different seed solutions, auto-Bäcklund transformation, the Cole-Hopf transformation and a functional separation exact solution containing two low dimensional arbitrary functions are obtained for the equation in question. Some interesting localized coherent structures are given and their interaction properties are numerically studied. Some new nonlinear phenomena are reported.
\end{abstract}

PACS: 05.45.Yv, 02.30.Jr, 02.30.Ik

\section{Introduction}

The solution of the form

$$
u=f(w), \quad w=a(x)+b(t),
$$

for a given nonlinear partial differential equation (PDE), say, in two independent variables, is called the functional separation solution [1]. The classical additive separable solution $u=a(x)+b(t)$, or product separable solution $u=a(x) b(t)$, are particular cases of functional separation. A simple particular case of (1) is the travelling wave solution, which is very well understood and hence omitted. There are a few papers coping with the direct method based on the general ansatz (1) [2-5] to consider the functional separable solutions of nonlinear PDEs. From the point of view of symmetry group, two methods have been proposed to study the compatibility of the ansatz (1) and the equation in question. One is the nonclassical method [6] where three different invariant surface conditions are used to characterize the functional separable solutions. The other is the generalized conditional symmetry approach based on the ansatz (1) [7]. The idea of functional separable is further developed by considering a $(3+1)$-dimensional Burgers equation of the form

$$
\begin{aligned}
& u_{t}+2 u u_{y}+2 v u_{x}+2 w u_{z}+u_{x x}+u_{y y}+u_{z z}=0, \\
& u_{x}=v_{y}, \quad u_{z}=w_{y},
\end{aligned}
$$

which has been given by Ying and Lou [8], and then reobtained by Dai et al. [9] from the invertible transformation of the heat conduction equation. Although its physical applications has not been found, the exact solution struc-

\footnotetext{
* e-mail: yanzepeng@163.com
}

ture of Eq. (2) is studied by several authors [10]. Their results indicate that Eq. (2) is a solitary equation. In this paper, we get an auto-Bäcklund transformation and the Cole-Hopf transformation of Eq. (2) by the singular manifold method [11, 12]. Under the help of the functional separation method, an exact solution containing two arbitrary functions is obtained for the equation in question. On the basis of the exact solution, some interesting localized coherent structures are given and their interaction properties are numerically studied.

\section{Some properties of the solution for Eq. (2)}

According to the singular manifold method [11, 12], we truncate the Painlevé expansion of Eq. (2) at the constant level term

$$
\begin{aligned}
& u=\varphi^{-1} u_{0}+u_{1}, \\
& v=\varphi^{-1} v_{0}+v_{1}, \\
& w=\varphi^{-1} w_{0}+w_{1},
\end{aligned}
$$

where $\varphi$ is the singular manifold, and $\left\{u_{1}, v_{1}, w_{1}\right\}$ is an arbitrary seed solution of Eq. (2). Substituting Eq. (3) into Eq. (2) and equating the coefficients of like powers of $\varphi$ yield

$$
u_{0}=\varphi_{y}, \quad v_{0}=\varphi_{x}, \quad w_{0}=\varphi_{z},
$$

where $\varphi$ satisfies the equation

$$
\begin{gathered}
\varphi_{t}+2 u_{1} \varphi_{y}+2 v_{1} \varphi_{x}+2 w_{1} \varphi_{z} \\
+\varphi_{x x}+\varphi_{y y}+\varphi_{z z}=0,
\end{gathered}
$$

which is called the singular manifold equation. Equations (3), (4) and (5) constitute an auto-Bäcklund transformation for Eq. (2) in terms of the singular manifold $\varphi$. If we take $u_{1}=\varphi, v_{1}=\partial_{y}^{-1} \varphi_{x}, w_{1}=\partial_{y}^{-1} \varphi_{z}$, then 
$u=\frac{\varphi_{y}}{\varphi}+\varphi, \quad v=\frac{\varphi_{x}}{\varphi}+\partial_{y}^{-1} \varphi_{x}, \quad w=\frac{\varphi_{z}}{\varphi}+\partial_{y}^{-1} \varphi_{z}$,

with $\varphi$ satisfying

$$
\begin{aligned}
& \varphi_{t}+2 \varphi \varphi_{y}+2\left(\partial_{y}^{-1} \varphi_{x}\right) \varphi_{x}+2\left(\partial_{y}^{-1} \varphi_{z}\right) \varphi_{z} \\
& \quad+\varphi_{x x}+\varphi_{y y}+\varphi_{z z}=0 .
\end{aligned}
$$

Equations (6) and (7) are another auto-Bäcklund transformation for $\mathrm{Eq}$. (2). If we take $u_{1}=0, v_{1}=0, w_{1}=0$, the Cole-Hopf type transformation or hetero-Bäcklund transformation

$$
u=\frac{\varphi_{y}}{\varphi}, \quad v=\frac{\varphi_{x}}{\varphi}, \quad w=\frac{\varphi_{z}}{\varphi},
$$

where $\varphi$ satisfies

$$
\varphi_{t}+\varphi_{x x}+\varphi_{y y}+\varphi_{z z}=0,
$$

is obtained for $(3+1)$-dimensional Burgers Eq. (2). If we take

$$
u_{1}=u_{1}(y, t), \quad v_{1}=v_{1}(x, z, t), \quad w_{1}=w_{1}(x, z, t),
$$

with $u_{1}$ satisfying $(1+1)$-dimensional Burgers equation

$$
u_{1 t}+2 u_{1} u_{1 y}+u_{1 y y}=0,
$$

and $v_{1}$ and $w_{1}$ being arbitrary functions of indicated variables, Eqs. (3), (4), (5) and (11) with (10) constitute another new Bäcklund transformation for Eq. (2). Now, we solve the last new Bäcklund transformation. After careful analysis, a functional separation solution follows from Eq. (5)

$$
\varphi=f(y, t) g(x, z, t)+h(x, z, t),
$$

with $f$ satisfying the linear equation

$$
f_{t}+2 u_{1} f_{y}+f_{y y}=0,
$$

and $g, h$ being arbitrary functions of indicated variables, while $v_{1}$ and $w_{1}$ satisfy the following constraint condition

$$
\begin{aligned}
& 2 g_{x} v_{1}+2 g_{z} w_{1}=-\left(g_{t}+g_{x x}+g_{z z}\right), \\
& 2 h_{x} v_{1}+2 h_{z} w_{1}=-\left(h_{t}+h_{x x}+h_{z z}\right) .
\end{aligned}
$$

Thus, we obtain an exact solution with two arbitrary functions for the Burgers Eq. (2)

$$
\begin{aligned}
& u=\frac{f_{y} g}{f g+h}+u_{1}, \\
& v=\frac{f g_{x}+h_{x}}{f g+h}+v_{1}, \\
& w=\frac{f g_{z}+h_{z}}{f g+h}+w_{1},
\end{aligned}
$$

where $f, g, h, u_{1}, v_{1}$, and $w_{1}$ are some functions as mentioned in Eqs. (10)-(14).

\section{Localized coherent structures and their interaction properties of Eq. (2)}

The solution generated this way involves two arbitrary functions of space and time variables without any restriction. This implies that we can study a large diversity of solution structures for the $(3+1)$-dimensional Burgers
Eq. (2) by selecting appropriately these arbitrary functions in Eq. (15). It is necessary to point out that the $(3+1)$-dimensional Burgers Eq. (2) possesses some special types of localized coherent structures, say, for the following potential field:

$$
\begin{aligned}
U & \equiv u_{x}=\frac{f_{y}\left(g_{x} h-g h_{x}\right)}{(f g+h)^{2}} \\
& \equiv(\ln (f(y, t) g(x, z, t)+h(x, z, t)))_{x y},
\end{aligned}
$$

rather than the physical field $u$ or the potential fields $v$ and $w$ themselves. In what follows, several interesting cases are considered.

First, we take the solution of Eq. (11) $u_{1}=C$, an arbitrary constant. Equation (13) has the solution

$$
\begin{aligned}
f & =\exp \left(l_{1} y-\left(2 C l_{1}+l_{1}^{2}\right) t\right) \\
& +\exp \left(l_{2} y-\left(2 C l_{2}+l_{2}^{2}\right) t\right) .
\end{aligned}
$$

Case 1. $g=1, h=\tanh \left(k_{1} x+n_{1} z-\omega_{1} t\right)+\tanh \left(k_{2} x+\right.$ $\left.n_{2} z-\omega_{2} t\right)+A$. Let us note that $k_{i}, l_{i}, n_{i}, \omega_{i}$ and etc. are all arbitrary constants throughout the paper, unless otherwise stated. It follows from Eq. (16) that

$$
\begin{aligned}
U & =\left[\operatorname { l n } \left(\exp \left(l_{1} y-\left(2 C l_{1}+l_{1}^{2}\right) t\right)\right.\right. \\
& +\exp \left(l_{2} y-\left(2 C l_{2}+l_{2}^{2}\right) t\right) \\
& +\tanh \left(k_{1} x+n_{1} z-\omega_{1} t\right) \\
& \left.\left.+\tanh \left(k_{2} x+n_{2} z-\omega_{2} t\right)+A\right)\right]_{x y} .
\end{aligned}
$$

In order to see the structure of Eq. (18), we draw its time evolution plots at $z=0$, and $t=-5,0,5$, respectively in Fig. 1. The parameters are $k_{1}=1, k_{2}=2, l_{1}=1, l_{2}=2$, $n_{1}=1, n_{2}=2, \omega_{1}=1, \omega_{2}=-1, A=4$ and $C=1$, which are valid throughout the figures, unless otherwise explained. It is easily found that this is a two-dromion-like structure and their interaction is inelastic.

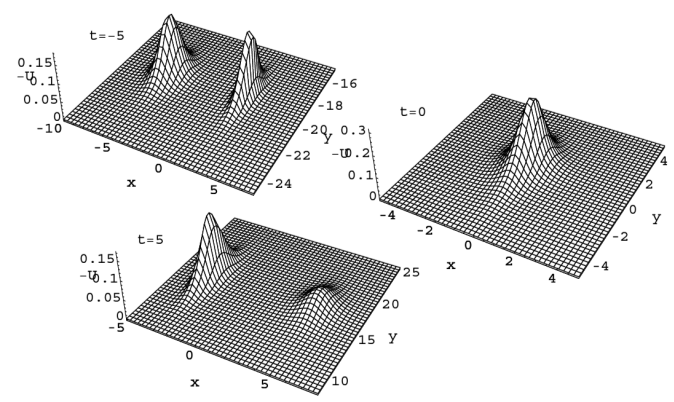

Fig. 1. The evolution of two dromions-like for Eq. (18).

Case 2. $g=1, h=\operatorname{sech}\left(k_{1} x+n_{1} z-\omega_{1} t\right)+\operatorname{sech}\left(k_{2} x+\right.$ $\left.n_{2} z-\omega_{2} t\right)+A$. From Eq. (16), one has

$$
\begin{aligned}
U & =\left[\operatorname { l n } \left(\exp \left(l_{1} y-\left(2 C l_{1}+l_{1}^{2}\right) t\right)\right.\right. \\
& +\exp \left(l_{2} y-\left(2 C l_{2}+l_{2}^{2}\right) t\right) \\
& +\operatorname{sech}\left(k_{1} x+n_{1} z-\omega_{1} t\right) \\
& \left.\left.+\operatorname{sech}\left(k_{2} x+n_{2} z-\omega_{2} t\right)+A\right)\right]_{x y},
\end{aligned}
$$

whose time evolution is depicted in Fig. 2. The four 
dromions-like solutions are divided into two groups, and each group is two. Its evolution graphs show the nonelastic property of interactions with new features. Two groups are all inelastic after interaction! In a dispersive long wave system [13], we find a multi-dromion-like structure solution whose interaction feature is that the one is elastic and the other is nonelastic after interaction of two groups. In a $(2+1)$-dimensional Burgers [14], a multi-dromion-like structure solution possesses the feature that two groups are all elastic after interaction.

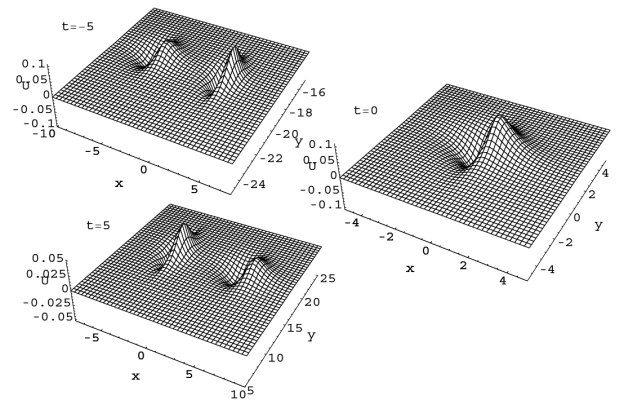

Fig. 2. The evolution of four dromions-like for Eq. (19).

Case 3. $g=1, h=\tanh \left(\tanh k_{1} x+n_{1} z-\omega_{1} t\right)+$ $\operatorname{sech}\left(\tanh k_{2} x+n_{2} z-\omega_{2} t\right)+A$.

In this case, we obtain

$$
\begin{aligned}
U & =\left[\operatorname { l n } \left(\exp \left(l_{1} y-\left(2 C l_{1}+l_{1}^{2}\right) t\right)\right.\right. \\
& +\exp \left(l_{2} y-\left(2 C l_{2}+l_{2}^{2}\right) t\right) \\
& +\tanh \left(\tanh k_{1} x+n_{1} z-\omega_{1} t\right) \\
& \left.\left.+\operatorname{sech}\left(\tanh k_{2} x+n_{2} z-\omega_{2} t\right)+A\right)\right]_{x y},
\end{aligned}
$$

whose evolution is drawn in Fig. 3. From the figures one

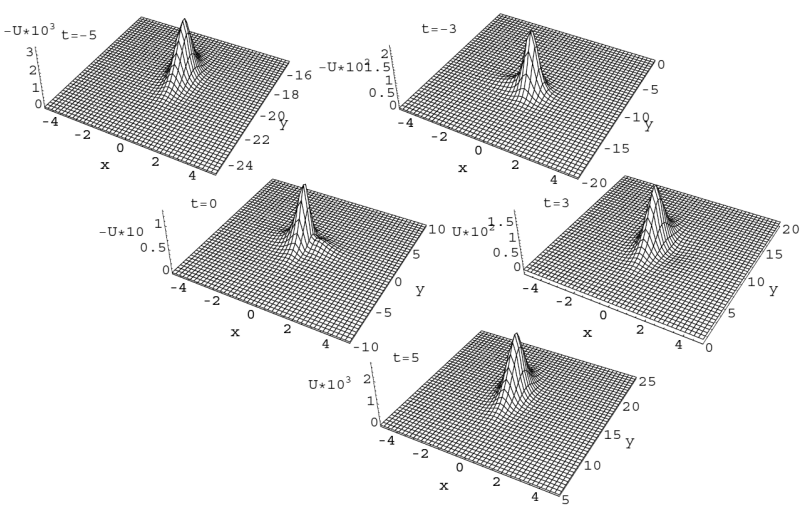

Fig. 3. The evolution graphs of (20) at $z=0$.

can clearly see the process of creation and annihilation of the dromion-like structure. Let us note that when $t>0$, the direction of dromion-like structure is in opposition to that of $t \leq 0$. In creation and annihilation phenomenon of the dromion-like structure for a Melnikov equation [15] and a restricted dispersive long wave system [16], the

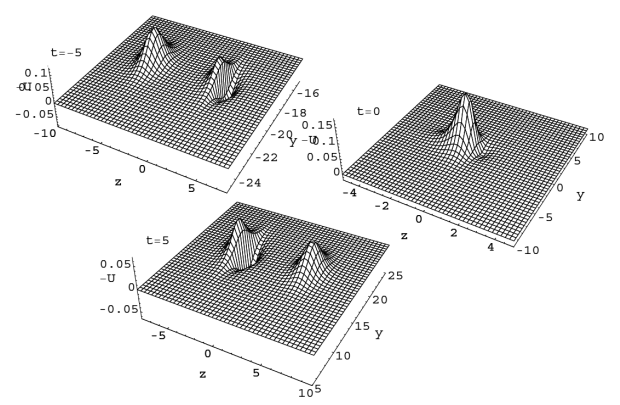

Fig. 4. The evolution of three dromions-like for Eq. (20) at $x=0$.

direction of dromion-like structure is unchanged. It is interesting to note that Eq. (20) at $x=0$ is a three-dromion-like structure. In order to see this, its evolution graphs are plotted in Fig. 4. The interaction of three dromions-like is inelastic.

Case 4. $g=\operatorname{sech}\left(k_{1} x+n_{1} z-\omega_{1} t\right)+\operatorname{sech}\left(k_{2} x+n_{2} z-\right.$ $\left.\omega_{2} t\right), h=A$. It follows from Eq. (16) that

$$
\begin{aligned}
U & =\left[\operatorname { l n } \left(\left(\exp \left(l_{1} y-\left(2 C l_{1}+l_{1}^{2}\right) t\right)\right.\right.\right. \\
& \left.+\exp \left(l_{2} y-\left(2 C l_{2}+l_{2}^{2}\right) t\right)\right) \\
& \times\left(\operatorname{sech}\left(k_{1} x+n_{1} z-\omega_{1} t\right)\right. \\
& \left.\left.\left.+\operatorname{sech}\left(k_{2} x+n_{2} z-\omega_{2} t\right)\right)+A\right)\right]_{x y},
\end{aligned}
$$

which is a two-dromiom-solitoff-like structure, and its evolution is shown in Fig. 5, which indicates that their interaction is inelastic because their directions are all changed after interaction.

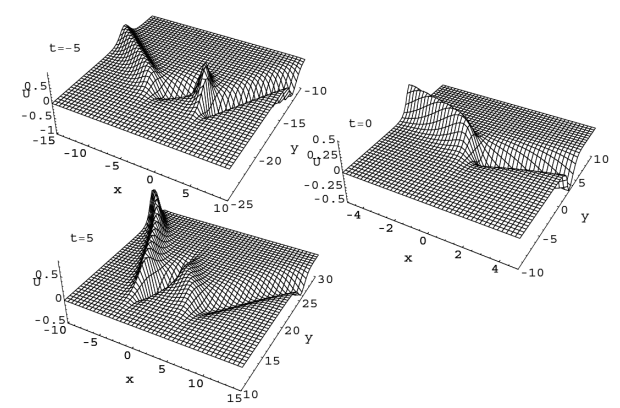

Fig. 5. The evolution of Eq. (21).

Case 5. $g=\exp (\tanh (k x+n z-\omega t)), h=$ $\exp \left(\tanh \left(k_{1} x+n_{1} z-\omega_{1} t\right)\right)+\exp \left(\tanh \left(k_{2} x+n_{2} z-\omega_{2} t\right)\right)+$ $A$. From Eq. (16), we have

$$
\begin{aligned}
U & =\left[\operatorname { l n } \left(\left(\exp \left(l_{1} y-\left(2 C l_{1}+l_{1}^{2}\right) t\right)\right.\right.\right. \\
& \left.+\exp \left(l_{2} y-\left(2 C l_{2}+l_{2}^{2}\right) t\right)\right) \\
& \times \exp (\tanh (k x+n z-\omega t)) \\
& +\exp \left(\tanh \left(k_{1} x+n_{1} z-\omega_{1} t\right)\right) \\
& \left.\left.+\exp \left(\tanh \left(k_{2} x+n_{2} z-\omega_{2} t\right)\right)+A\right)\right]_{x y} .
\end{aligned}
$$

This is a two-dromion-like structure (with opposite direc- 


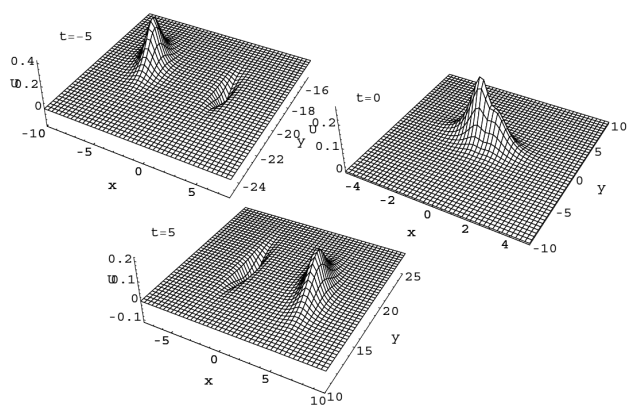

Fig. 6. The evolution of Eq. (22).

tion) structure, whose interaction is made out in Fig. 6 with $k=1, n=2$ and $\omega=1$. We see that their amplitudes are changed after interaction.

Case 6. $g=\exp (\tanh (k x+n z)-\omega t), \quad h=$ $\exp \left(\tanh \left(k_{1} x+n_{1} z\right)-\omega_{1} t\right)+\exp \left(\tanh \left(k_{2} x+n_{2} z\right)-\omega_{2} t\right)+$ A.

From Eq. (16), one has

$$
\begin{aligned}
U & =\left[\operatorname { l n } \left(\left(\exp \left(l_{1} y-\left(2 C l_{1}+l_{1}^{2}\right) t\right)\right.\right.\right. \\
& \left.+\exp \left(l_{2} y-\left(2 C l_{2}+l_{2}^{2}\right) t\right)\right) \\
& \times \exp (\tanh (k x+n z)-\omega t) \\
& +\exp \left(\tanh \left(k_{1} x+n_{1} z\right)-\omega_{1} t\right) \\
& \left.\left.+\exp \left(\tanh \left(k_{2} x+n_{2} z\right)-\omega_{2} t\right)+A\right)\right]_{x y},
\end{aligned}
$$

whose time evolution is accounted for in Fig. 7. From these figures, we find that two nonlinear phenomena have taken place. When $t<0$, the dromion-like structure creates because its amplitude is increasing with the time. When $t \geq 0$, one dromion-like structure is fissioned into three, which propagate then steadily. The combination phenomenon of creation and fission in nonlinear evolution equation has not yet been reported previously in the literature to our knowledge.

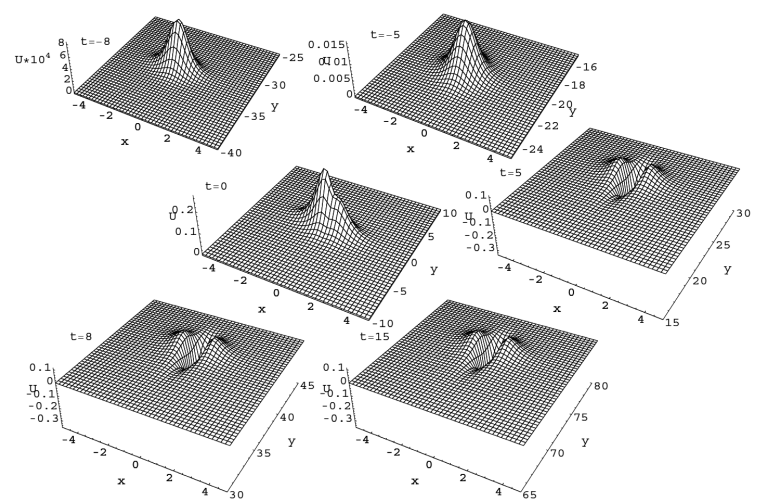

Fig. 7. The evolution graphs of (23).

Now, we take the solution of Eq. (11) $u_{1}=l \tanh (l y-$ $\lambda t)+\lambda /(2 l)$ with $l \neq 0, \lambda$ being two arbitrary constants. Then Eq. (13) has the solution

$$
f=a \tanh (l y-\lambda t)+b,
$$

where $a$ and $b$ are arbitrary constants. Several interesting cases are considered.

Case 7. $g=\tanh (k x+n z-\omega t)+c, h=\tanh \left(k_{1} x+\right.$ $\left.n_{1} z-\omega_{1} t\right)+\tanh \left(k_{2} x+n_{2} z-\omega_{2} t\right)+A$.

It follows from Eq. (16) that

$$
\begin{aligned}
U & =[\ln ((a \tanh (l y-\lambda t)+b) \\
& \times(\tanh (k x+n z-\omega t)+c) \\
& +\tanh \left(k_{1} x+n_{1} z-\omega_{1} t\right) \\
& \left.\left.+\tanh \left(k_{2} x+n_{2} z-\omega_{2} t\right)+A\right)\right]_{x y} .
\end{aligned}
$$

Although the parameters $a, b, c$, and $A$ are arbitrary, they should be taken under the condition that $U$ has no singularity. The evolution of Eq. (25) is shown in Fig. 8 with the parameters $a=1, b=2, c=2, l=2$ and $\lambda=-1$, which are valid in the following figures, unless otherwise explained. It is found from the graphs that two dromions-like fissioned into three first and then fused two.

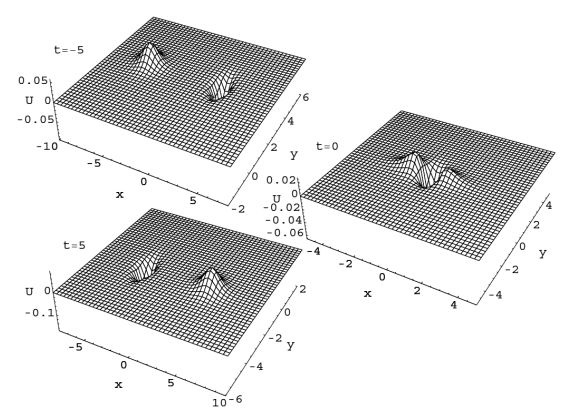

Fig. 8. The evolution of Eq. (25).

Case 8. $g=\tanh (k x+n z-\omega t)+c, h=\tanh \left(\tanh k_{1} x+\right.$ $\left.\tanh n_{1} z-\omega_{1} t\right)+\tanh \left(\tanh k_{2} x+\tanh n_{2} z-\omega_{2} t\right)+A$.

In this case, we have

$$
\begin{aligned}
U & =[\ln ((a \tanh (l y-\lambda t)+b) \\
& \times(\tanh (k x+n z-\omega t)+c) \\
& +\tanh \left(\tanh k_{1} x+\tanh n_{1} z-\omega_{1} t\right) \\
& \left.\left.+\tanh \left(\tanh k_{2} x+\tanh n_{2} z-\omega_{2} t\right)+A\right)\right]_{x y},
\end{aligned}
$$

whose evolution is explained by Fig. 9. One can easily see that one dromion-like fissioned into three first and then fused one. It is interesting to see that in opposition to case 7 , the dromion is identic before and after interaction.

Case 9. $g=\tanh (k x+n z-\omega t)+c, h=\tanh \left(\operatorname{sech} k_{1} x+\right.$ $\left.\operatorname{sech} n_{1} z-\omega_{1} t\right)+\tanh \left(\tanh k_{2} x+\tanh n_{2} z-\omega_{2} t\right)+A$.

Now, one has

$$
\begin{aligned}
U & =[\ln ((a \tanh (l y-\lambda t)+b) \\
& \times(\tanh (k x+n z-\omega t)+c) \\
& +\tanh \left(\operatorname{sech} k_{1} x+\operatorname{sech} n_{1} z-\omega_{1} t\right) \\
& \left.\left.+\tanh \left(\tanh k_{2} x+\tanh n_{2} z-\omega_{2} t\right)+A\right)\right]_{x y} .
\end{aligned}
$$

Its evolution property is displayed in Fig. 10, which indicates that one dromion-like structure fissioned into two 


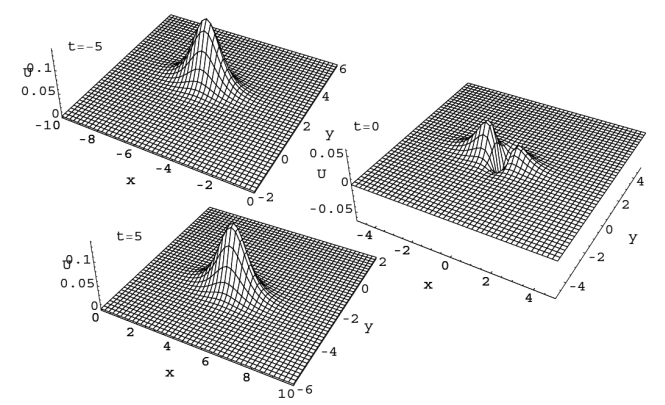

Fig. 9. The evolution of Eq. (26).

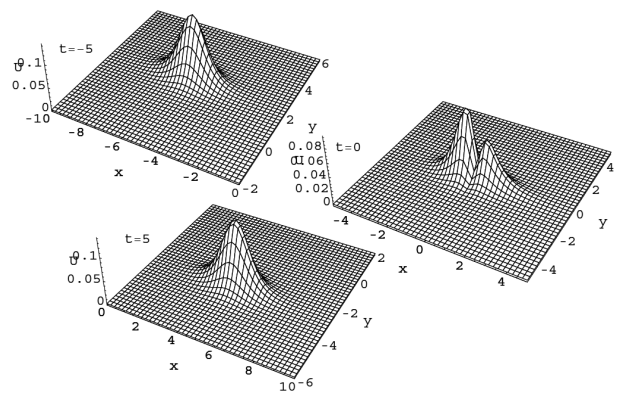

Fig. 10. The evolution of Eq. (27).

first and then fused one, and the dromion is identic before and after interaction.

Case 10. $g=\tanh (k x+n z-\omega t)+c, h=$ $\tanh \left(\operatorname{sech} k_{1} x+\operatorname{sech} n_{1} z-\omega_{1} t\right)+\tanh \left(\operatorname{sech} k_{2} x+\right.$ $\left.\operatorname{sech} n_{2} z-\omega_{2} t\right)+A$. It follows from Eq. (16) that

$$
\begin{aligned}
U & =[\ln ((a \tanh (l y-\lambda t)+b) \\
& \times(\tanh (k x+n z-\omega t)+c) \\
& +\tanh \left(\operatorname{sech} k_{1} x+\operatorname{sech} n_{1} z-\omega_{1} t\right) \\
& \left.\left.+\tanh \left(\operatorname{sech} k_{2} x+\operatorname{sech} n_{2} z-\omega_{2} t\right)+A\right)\right]_{x y} .
\end{aligned}
$$

It is surprising that Eq. (28) is a single dromion-like structure and can propagate steadily. Its structure is the same as the first one of Fig. 10.

\section{Conclusion and discussion}

The property of the solution for the $(3+1)$-dimensional Burgers Eq. (2) has been in detail studied by using the singular manifold method. An auto-Bäcklund transformation, a Cole-Hopf type transformation and a functional separation exact solution containing two low dimensional arbitrary functions are obtained. On the basis of the exact solution, some interesting localized coherent structures are revealed. Some nonlinear phenomena, such as elastic and inelastic interaction, creation and annihilation phenomenon, fission and fusion phenomenon, with new properties are detected. It is worth studying further whether Eq. (2) has solutions other than Eq. (15).

\section{Acknowledgments}

This work is supported by Research Fund for the Doctoral Program of Higher Education of China (No. 20070486094).

\section{References}

[1] W. Miller, Jr., in: Symmetries and Nonlinear Phenomena, Eds. D. Levi, P. Winternitz, World Sci., London 1989.

[2] E.G. Kalnins, W. Miller, J. Math. Phys. 26, 1560 (1985).

[3] O.V. Kaptsov, Nonlinear Anal. 19, 753 (1992).

[4] W. Miller, Jr., L.A. Rubel, J. Phys. A 26, 1901 (1993).

[5] R.Z. Zhdanov, J. Phys. A 27, L291 (1994).

[6] E. Pucci, G. Saccomandi, Physica D 139, 28 (2000).

[7] C.Z. Qu, S.L. Zhang, R.C. Liu, Physica D 144, 97 (2000).

[8] J.P. Ying, S.Y. Lou, Chin. Phys. Lett. 20, 1448 (2003).

[9] C.Q. Dai, C.J. Yan, J.F. Zhang, Commun. Theor. Phys. 46, 389 (2006).

[10] S.F. Shen, Z.L. Pan, J.F. Zhang, Commun. Theor. Phys. 42, 49 (2004).

[11] J. Wess, J. Math. Phys. 26, 258 (1985).

[12] Y.Z. Peng, Prog. Theor. Phys. 113, 927 (2005).

[13] Y.Z. Peng, E.V. Krishnan, Rep. Math. Phys. 56, 367 (2005).

[14] Y.Z. Peng, E. Yomba, Appl. Math. Comput. 183, 61 (2006).

[15] Y.Z. Peng, Z. Naturforsch. A 61, 253 (2006).

[16] Y.Z. Peng, Rep. Math. Phys. 60, 259 (2007). 